\title{
On the direct correlation between gamma-rays and PeV neutrinos from blazars
}

\author{
Shan Gao* \\ Deutsches Elektronen-Synchrotron (DESY), Platanenallee 6, D-15738 Zeuthen, Germany \\ E-mail: shan.gaodesy.de \\ Martin Pohl \\ Deutsches Elektronen-Synchrotron (DESY), Platanenallee 6, D-15738 Zeuthen, Germany \\ E-mail: martin.pohledesy.de \\ Walter Winter \\ Deutsches Elektronen-Synchrotron (DESY), Platanenallee 6, D-15738 Zeuthen, Germany \\ E-mail: walter.winteredesy.de
}

\begin{abstract}
We study the frequently used assumption in multi-messenger astrophysics that the gamma-ray and neutrino fluxes are directly connected because they are assumed to be produced by the same photohadronic production chain. An interesting candidate source for this test is the flat-spectrum radio quasar PKS B1424-418, which recently called attention of a potential correlation between an IceCube PeV-neutrino event and its burst phase. We simulate both the multi-waveband photon and the neutrino emission from this source using a self-consistent radiation model. We demonstrate that a simple hadronic model cannot adequately describe the spectral energy distribution for this source, but a lepto-hadronic model with sub-dominant hadronic component can reproduce the multi-waveband photon spectrum observed during various activity phases of the blazar. As a conclusion, up to about 0.3 neutrino events may coincide with the burst, which implies that the leptonic contribution dominates in the relevant energy band. We also demonstrate that the timewise correlation between the neutrino event and burst phase is weak.
\end{abstract}

35th International Cosmic Ray Conference - ICRC2017

12-20 July, 2017

Bexco, Busan, Korea

* Speaker. 


\section{Introduction}

The existence of TeV-PeV astrophysical neutrinos flux by IceCube (IceCube Collaboration, 2013) is perhaps one of the most exciting breakthroughs recently. However, the nature of their sources remains unknown. Among the cosmic accelerators are supernova remnants, Gamma-ray Bursts, active galactic nuclei (AGN), star-burst galaxies, galaxy clusters and mergers, etc., which are all promising source candidates. Blazars, a subclass of AGNs with a relativistic jet pointing towards the line of sight, significantly contribute to the diffuse (extragalactic) $\gamma$-ray background. If these $\gamma$-rays are derived from proton interactions, the energy budget will be sufficient to account for the IceCube neutrino by the same process.

Blazars typically exhibit a two-hump structure in their spectral energy distribution (SED) (Ghisellini et al., 2017), the first of which has been successfully reproduced in theory by the electron-synchrotron emission, where the second hump can be explained by the emission from either the leptonic inverse Compton scattering (IC) or the secondaries from hadronic interactions. In the simplest case, it is frequently assumed that in $p \gamma$ interactions the $\gamma$-ray luminosity $L_{\gamma}$ from $\pi^{0}$ decays is roughly the same as the neutrino luminosity $L_{v}$ from $\pi^{ \pm}$decays, which means that the neutrno and $\gamma$-ray fluxes are directly correlated. Note, however, that even in hadronic scenarios, the $\gamma$-rays from $\pi^{0}$ decays may be absorbed quickly in situ leading to a electromagnetic cascade down to lower energies. In other scenarios, the leading contribution to the second hump comes from the synchrotron or IC emission by $e^{ \pm}$pairs from either Bethe-Heitler process or $\pi^{ \pm}$ decays. In addition, in some blazars, it is possible that the second hump may be explained only via leptonic-dominated scenarios. Therefore, the main motivation of this work (for a full version of refereed paper, see (Gao et al., 2016)) is to study this $L_{v} \sim L_{\gamma}$ correlation in blazars.

One interesting test case for the direct neutrino- $\gamma$-ray correlation is the flat-spectrum radio quasar (FSRQ) PKS B1424-418. In (Kadler et al., 2016) (K16), a positional and temporal coincidence between the 2-PeV neutrino event (IceCube event 35, IC35, or "big bird") and a burst of PKS B1424-418 was reported. After analyzing the $\gamma$-ray fluences from blazars in the positionaluncertainty region of IC35 as well as the diffuse $\gamma$-ray emission, it was concluded that the burst of PKS B1424-418 had sufficient energy to account for the IceCube event, while the probability of a chance coincidence was around 5\%. An important ingredient was the assumption that the SED in that respective energy range were of hadronic origin, implying the $L_{v} \sim L_{\gamma}$ direct correlation. We study if this assumption can be maintained in a self-consistent ansatz. Note that while we focus on one astrophysical object in this study, our conclusions will be more profound, as this direct correlation is widely used in multi-messenger analyses.

\section{General Analysis and Models}

In Table. 1 we broadly list possible models and give generic constraints based on the characteristics of the SED for the source. The conclusions are derived using analytical and semi-analytical methods.

While both the leptonic and the hadronic models have successfully explained the SED of a number of blazars, for example Mrk 421, 3C 279 etc., the unique combination of the SED with the PeV-neutrino information places stringent limits on the model of PKS B1424-418. After analytical 


\begin{tabular}{|c|c|c|c|}
\hline & $\begin{array}{l}\text { First peak } \\
(\mathrm{eV}-\mathrm{keV})\end{array}$ & $\begin{array}{l}\text { Middle range } \\
(\mathrm{keV}-\mathrm{MeV})\end{array}$ & $\begin{array}{l}\text { Second peak } \\
(\mathrm{MeV}-\mathrm{TeV})\end{array}$ \\
\hline $\begin{array}{c}\text { SSC } \\
\text { (Pure leptonic) }\end{array}$ & $\begin{array}{c}\mathbf{L} \\
\text { Primary } e^{-} \text {synchrotron }\end{array}$ & $\begin{array}{c}\mathbf{L} \\
\mathrm{SSC}\end{array}$ & $\begin{array}{c}\mathbf{L} \\
\mathrm{SSC}\end{array}$ \\
\hline $\begin{array}{c}\text { LH-SSC } \\
\text { (Lepto-hadronic) }\end{array}$ & $\begin{array}{c}\mathbf{L} \\
\text { Primary } e^{-} \text {synchrotron }\end{array}$ & $\begin{array}{c}\mathbf{H} \\
\text { Secondary leptonic }\end{array}$ & $\begin{array}{c}\mathbf{L} \\
\text { SSC by primary } e^{-}\end{array}$ \\
\hline $\begin{array}{c}\text { LH- } \pi \\
\text { (Lepto-hadronic) }\end{array}$ & $\begin{array}{c}\mathbf{L} \\
\text { Primary } e^{-} \text {synchrotron }\end{array}$ & $\begin{array}{c}\mathbf{H} \\
\text { Secondary leptonic }\end{array}$ & $\begin{array}{l}\mathbf{H} \\
\text { Secondary leptonic or } \gamma- \\
\text { rays from direct } \pi^{0} \text { decay }\end{array}$ \\
\hline $\begin{array}{c}\text { LH-psyn } \\
\text { (Proton synchrotron) }\end{array}$ & $\begin{array}{c}\mathbf{L} \\
\text { Primary } e^{-} \text {synchrotron }\end{array}$ & \begin{tabular}{l}
\multicolumn{1}{c}{$\mathbf{H}$} \\
Proton synchrotron \\
or secondary lep- \\
tonic
\end{tabular} & $\begin{array}{c}\mathbf{H} \\
\text { Proton synchrotron }\end{array}$ \\
\hline $\begin{array}{c}\text { Proton } \\
\text { (Pure hadronic) }\end{array}$ & $\begin{array}{c}\mathbf{H} \\
\text { Proton synchrotron }\end{array}$ & $\begin{array}{c}\mathbf{H} \\
\text { Secondary leptonic }\end{array}$ & $\begin{array}{l}\mathbf{H} \\
\text { Secondary leptonic or } \gamma- \\
\text { rays from direct } \pi^{0} \text { decay }\end{array}$ \\
\hline
\end{tabular}

Table 1: List of models. In the table, "L"="Leptonic" and "H"="Hadronic". "LH" is the abbreviation for "lepto-hadronic", which is a mixture of leptonic and hadronic components.

and semi-analytical calculations we demonstrate that neither the lepto-hadronic (LH $\pi$, LH-psyn) nor the purely hadronic proton model can simultaneously explain the SED and the PeV-neutrino event, leaving the SSC and LH-SSC models of the SED as the only viable contenders. Analytical arguments also give useful constraints on the parameter space. See (Gao et al., 2016) for full details.

\section{Numerical SED Model and Consequences for the Neutrino Production}

For an accurate modeling of SED and neutrino emission from PKS B1424-418, we simulate time-dependent particle spectra for $e^{ \pm}, p, n, \gamma$, and $v_{\alpha}$ ( $\alpha$ denotes the neutrino flavor) by numerically solving the time-dependent differential-integral kinematic-equation system in the energy space $\gamma$, for all the particle species mentioned above:

$$
\partial_{t} n(\gamma, t)=-\partial_{\gamma}\left\{\dot{\gamma}(\gamma, t) n(\gamma, t)-\partial_{\gamma}[D(\gamma, t) n(\gamma, t)] / 2\right\}-\alpha(\gamma, t) n(\gamma, t)+Q(\gamma, t)
$$

where $n(\gamma) \equiv d^{2} N / d \gamma d V$ is the differential number density of the particle species. In the equation above, the source term $Q$ models the injection, emission or generation of a new particle after an interaction, or re-distribution or re-injection of the same particle after scattering. The sink term $\alpha$ models the escape of the particle from the blob, decay, annihilation and disappearance due to an interaction. The differential terms $\dot{\gamma}(\gamma, t)$ and $D(\gamma, t)$ account for the particle cooling and diffusion effect in the momentum space due to synchrotron radiation, Thomson scattering and Bethe-Heitler process, where we apply the "continuous-loss" approximation.

The rates and redistribution functions are described by physics, where we consider synchrotron, inverse Compton, pair production and annihilation, photo-hadronic $(p \gamma)$ interaction and BetheHeitler (photo-pair) process. See (Hümmer et al., 2010) (H10) for the simplified $p \gamma$ interaction 


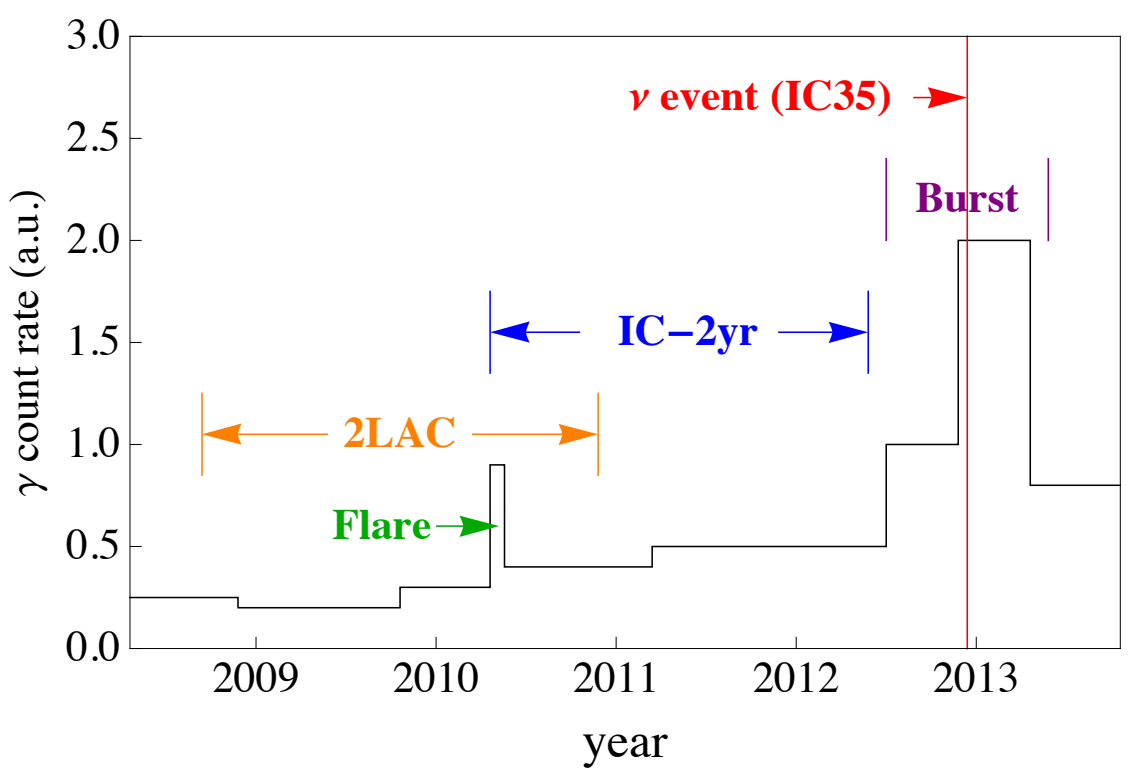

Figure 1: An illustration of the $\gamma$-ray count rate as a function of time together with a definition of the 2LAC, IC-2yr, and Burst phases of PKS B1424-418. Note that the count rate is meant for illustrative purposes only and does not accurately reflect the data (see Figure 1 of K16 for the bi-weekly binned $\gamma$-ray light curve). Also note that K16 shows the SED from the IceCube 3-year phase, which overlaps with the Burst phase. The IC-2yr SED is constructed and provided by the authors of K16, which is not shown in their paper.

model which we apply in this paper. We use the finite-difference method to solve the equation numerically, on an evenly-spaced logarithmic grid in energy and a linear one in time, such as (Vurm \& Poutanen, 2009).

The dynamical SEDs of PKS B1424-418 reported in K16 are categorized into four phases: (1) flare in 2010, lasting about 1 month; (2) 2LAC phase, from 2008.8 to 2010.9; (3) IC-2yr period, from 2010.5 to 2012.5, the first two years of IceCube observation; (4) Burst phase, from 2012.6 to 2013.3, when the source experienced a long-lasting high-flux phase in $\gamma$-rays. Fig. 1 provides a visual timeline including the time-averaged $\mathrm{GeV}$-band $\gamma$-ray flux. A 2-PeV neutrino event in IceCube (IC35, also dubbed "big bird") was observed on Dec. 4, 2012, during phase 4, with a position consistent with that of PKS B1424-418. The SEDs shown in K16 are based on timeaveraged spectra from each phase. In this paper, the flare phase is ignored since its duration was too short to result in a neutrino event.

For each parameter-space simulation, we perform independent optimizations. First, we adapt our model to the SED and find the best fit and confidence regions by calculating the reduced $\chi^{2}$ values. This SED best-fit is marked with the symbol "+". Then with the predicted neutrino spectrum from each simulation, we calculate the probability, $P_{010}\left(E_{\mathrm{p}, \max }, \eta_{\mathrm{b}}\right)$ to observe from PKS B1424-418 with IceCube 0,1, and 0 neutrino events, as indeed observed, in the (0.5 - 1.6), (1.6$2.4)$, and $>2.4 \mathrm{PeV}$ energy bands, respectively. The best adaption to the neutrino data without regard of the SED is referred to by the symbol " $\times$ ", and its fit probability is denoted as $P_{v, \max }$. The joint best-fit point, by maximizing the joint probability of the SED and neutrino fit, is marked with the symbol "*⿻丷木". 


\begin{tabular}{|c|c|c|c|}
\hline & Group & Symbol & Definition \\
\hline \multirow{3}{*}{ Parameters } & Global & $\begin{array}{c}R_{\text {blob }}^{\prime} \\
f_{\text {esc }} \\
\Gamma_{\text {bulk }}\end{array}$ & $\begin{array}{l}\text { Comoving radius of blob, fixed to } 7.5 \times 10^{17} \mathrm{~cm} \\
e^{ \pm} \text {and } p \text { escape fraction, fixed to } 1 / 10 \\
\text { bulk Lorentz factor of the blob fixed to } 35\end{array}$ \\
\hline & Leptonic & $\begin{array}{c}B^{\prime} \\
L_{\mathrm{e}, \mathrm{inj}} \\
\gamma_{\mathrm{e}, \min }^{\prime} \\
\gamma_{\mathrm{e}, \max }^{\prime} \\
\alpha_{\mathrm{e}, \mathrm{idx}}^{\prime}\end{array}$ & $\begin{array}{l}\text { Magnetic field strength, blob frame } \\
\text { Injection luminosity of primary } e^{-}, \mathrm{AGN} \text { frame } \\
\text { Minimum Lorentz factor of primary } e^{-}, \text {blob frame } \\
\text { Maximum Lorentz factor of primary } e^{-}, \text {blob frame } \\
\text { Power law index of injected primary } e^{-}\end{array}$ \\
\hline & Hadronic & $\begin{array}{c}\alpha_{\mathrm{p}, \mathrm{idx}}^{\prime} \\
\eta_{\mathrm{b}} \\
E_{p, \max }^{o b} \\
\end{array}$ & $\begin{array}{l}\text { Power law index of injected protons, fixed to }-2.0 \\
\text { Luminosity ratio } p \text { to } e^{-} \text {at injection, i.e., } \eta_{\mathrm{b}} \equiv L_{\mathrm{p}, \text { inj }} / L_{\mathrm{e}, \text { inj }} \\
\text { Maximal energy of injected protons, observer frame }\end{array}$ \\
\hline Notations & $P_{0,1,0}\left(E_{\mathrm{p}}\right.$ & $\left.\max , \eta_{\mathrm{b}}\right)$ & $\begin{array}{l}\text { Probability to observe } 0,1,0 \text { neutrino events in the } 0.5-1.6 \mathrm{PeV} \text {, } \\
1.6-2.4 \mathrm{PeV} \text { and }>2.4 \mathrm{PeV} \text { bands in IceCube, respectively, as a } \\
\text { function of } E_{\mathrm{p}, \max } \text { and } \eta_{\mathrm{b}} \\
\text { Maximum value of } P_{0,1,0}\left(E_{\mathrm{p}, \max }, \eta_{\mathrm{b}}\right) \text { in the parameter space, } \\
\text { named as "neutrino best-fit" } \\
\text { Total neutrino luminosity, including all flavors } \\
\gamma \text {-ray luminosity integrated over the frequency band } 10^{18.1} \sim \\
10^{24.4} \mathrm{~Hz} \\
\text { SED best-fit mark } \\
\text { Neutrino best-fit mark, global } \\
\text { Joint best-fit mark, for SED and neutrino }\end{array}$ \\
\hline
\end{tabular}

Table 2: List of parameters and notations.

We find that both SED and neutrino fits independently prefer large baryonic loadings for the Burst phase, which may point towards a baryonically loaded burst. At the neutrino and joint bestfit points in Fig. $2(\times, *$, $)$, the associated proton maximum energies are around $10 \mathrm{PeV}$, which is consistent with the observed $\mathrm{PeV}$ neutrino event. However, the maximal proton flux at the neutrino best-fit $\times$ is in tension with the X-ray data, which we will demonstrate below.

We show the SED for the SED best-fit (+), the joint best-fit (*) and the neutrino best-fit $(\times)$ in the left panel of Fig. 2. We clearly observe that the SED is in tension with data in the X-ray energy range. On the other hand, the SED is described reasonably well for the other two fit points. Note that the radio data are fitted from the extended emission region.

We list the fractional contributions to the SED of different physical interactions for the joint best-fit case of Burst phase in the caption of Fig.3, where one can clearly read off that the SSC contribution dominates. We also show the neutrino-to- $\gamma$-ray ratio $L_{v} / L_{\gamma}$, which is order $5 \%$ for the models fitting the SED. These numbers are to be interpreted as an additional "theory" correction factor in addition to those included in K16. They reflect the fact that hadronic processes only dominate in a small portion of the energy range marked " $L_{\gamma}$ " in Fig.3. In K16, the predicted number of neutrino events in $1.0-2.0 \mathrm{PeV}$ bin is 1.6 , assuming that the entire second hump is generated from hadronic processes. From our model, this needs to be corrected by this factor of 

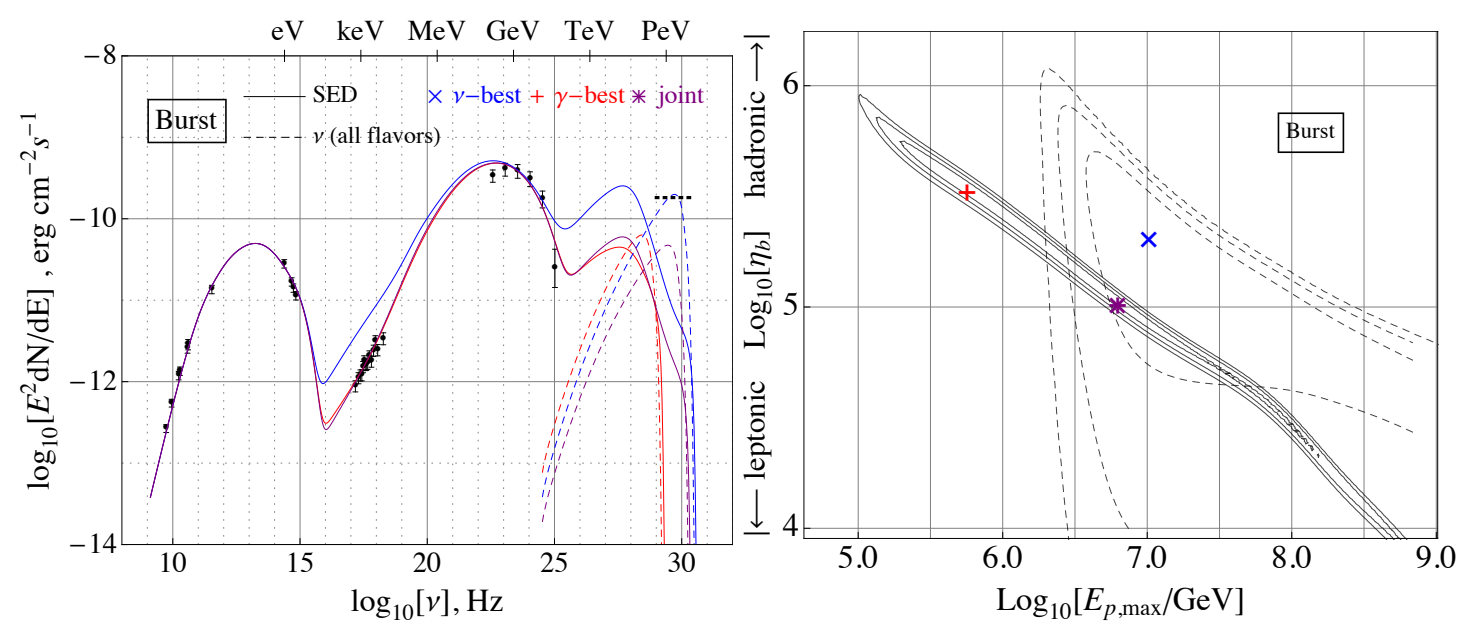

Figure 2: Right panel: fitting quality to SED and neutrino observations over the $E_{p, \max }$ and $\eta_{b}=L_{p, i n j} / L_{e, i n j}$ parameter-space for the Burst phase. Solid contours are the boundaries of $1 \sigma, 2 \sigma$, and $3 \sigma$ confidence regions for the SED fit, while dashed curves are iso-probability contours for $P / P_{v, \max }=0.32,0.05,0.003$ - see main text for details. The symbols,$+ \times$ and $*$ mark the best-fit parameters of the SED, neutrino and the joint fit, respectively. Left panel: the SED (solid) and neutrino (dashed) spectra, corresponding to the three marks on the right-panel. The horizontal dashed line is the neutrino flux level to observe one $\mathrm{PeV}$ event over this 9-month period. Data points are provided from authors of K16.

0.05 , arriving at $\sim 0.08$ events. Consistently, the neutrino spectrum from our numerical simulation (the joint best-fit case) predicts 0.094 events in IceCube within the same energy bin.

In order to draw a self-consistent picture of the AGN blazar over time, we independently fit the parameters for the three phases 2LAC, IC-2yr, and Burst in Fig. 1; The best-fit parameters for the $2 \mathrm{LAC}$ and IC-2yr phases turn out to be rather similar as the Burst phase, except that the required injection luminosities for electron and protons are lower. It is found that the correlation between the PeV neutrino event with the Burst phase, is weak, since the IC-2yr phase predicts up to (in the SED-allowed region) a higher probability $P_{0,1,0}=5.7 \%$ to produce the same observations in IceCube, than $P_{0,1,0}=3.2 \%$ in the Burst phase. The relative probability is therefore $P_{0,1,0}(\mathrm{IC}-2 \mathrm{yr}): P_{0,1,0}$ (Burst) $=1.6: 1$. Here $P_{0,1,0}$ is defined as the joint probability to observe $0,1,0$ neutrino events in the $0.5-1.6,1.6-2.4$ and $>2.4 \mathrm{PeV}$ energy bins, respectively, where in each bin, the expected number of neutrino events follows a Poisson distribution.

\section{Summary and conclusions}

A potential correlation between the PeV neutrino event "Big Bird" by IceCube in 2012 and the Burst phase of FSRQ PKS B1424-418 was reported by (Kadler et al., 2016) (K16). That analysis relies on the frequently used assumption $L_{\gamma} \approx L_{v}$. In this paper we have revisited this important relationship with a self-consistent one-zone emission model, with SEDs measured from three phases of the source. Those different SEDs can be interpreted in our results as variations on blazar properties over time. We found that the "conventional" hadronic model (high energy hump via hadronic processes; the "LH- $\pi$ " or "LH-psyn" model) does not work for this blazar, which is different from many other blazars, for which the high-energy hump of SED can be fully accounted 


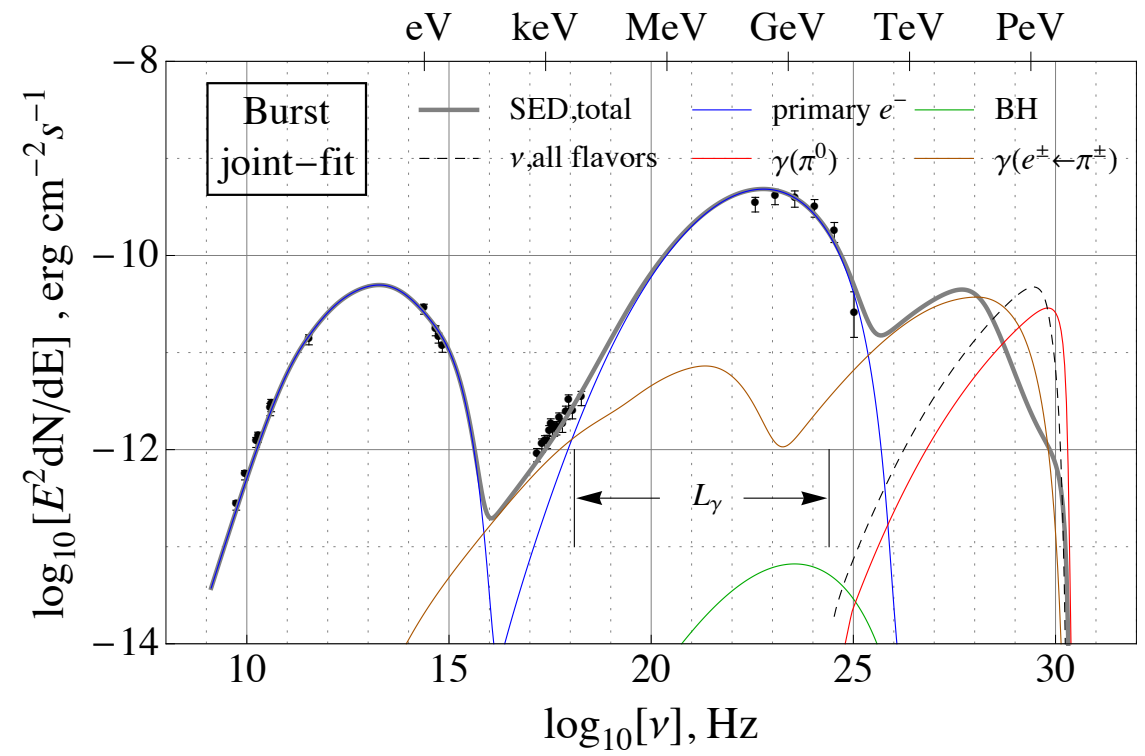

Figure 3: Components for the joint best-fit SED during burst phase. Gray: total SED; blue: emission from primary $e^{-}$; green: emission from pairs generated via Bethe-Heitler process; brown: $\gamma$-ray injection spectrum from $e^{ \pm}$pairs via $\pi^{ \pm}$decay. red: $\gamma$-ray injection spectrum from $\pi^{0}$ decay; blackdashed: neutrino, all flavors. The fractional contributions of these components to the $L_{\gamma}$ band, are $L_{s s c}=0.981, L_{\pi}=0.0185, L_{B H}=1.3 \times 10^{-4}$, respectively, where $L_{\gamma}$ is defined as the integrated luminosity between $10^{18.1} \sim 10^{24.4} \mathrm{~Hz}(5 \mathrm{keV}$ to $10 \mathrm{GeV})$. The bolometric neutrino to gamma-ray luminosity ratio is $L_{V} / L_{\gamma}=0.051$. The $\gamma \gamma$-absorption effect in the source becomes significant above $\sim 100 \mathrm{TeV}$, which is manifested in the suppression of the total SED in that energy band. The data points here are provided by authors of K16 (processed from data by Fermi-LAT, Swift-XRT/UVOT, SMARTS, and the LBA, etc; see the supplementary material for the data analysis methods in K16).

for by hadronic processes. Therefore, the leading contribution to the SED of PKS B1424-418 must be leptonic. We have, however, shown that a subdominant hadronic contribution can be present. In terms of neutrino production, it is therefore an interesting question how much baryonic loading can be tolerated, even in an SSC model, without affecting the shape of the SED.

We have found that the observed one neutrino event reported by K16 during the Burst phase is in tension with the SED fits, whereas up to $\sim 0.3$ above-PeV events are acceptable. The probability to produce 0,1 , and 0 neutrinos in the $0.5-1.6,1.6-2.4$, and $>2.4 \mathrm{PeV}$ bins, respectively, as observed by IceCube, is up to $5.7 \%$ in IC-2yr and 3.2\% in Burst phase. This suggests a chance coincidence of the observed PeV-event with the Burst phase of PKS B1424-418, since there is a even higher probability for it to occur during the IceCube 2-year observation than the Burst phase. Our predicted event rates are consistent with $\mathrm{K} 16$ if their expectation is corrected for small fraction of the photon energy flux in the second peak comes from hadronic processes. This means that an additional "theory correction factor" has to be applied if the gamma-ray and neutrino fluxes are to be correlated, which can only come from self-consistent models.

We have also demonstrated from independent simulations of the different phases of the blazar that a self-consistent time-dependent evolution picture can be drawn, meaning that the different phases can be described by similar parameters. We noted that the active state (Burst phase) can be 
achieved simply by increasing the injection rate of electrons and protons. It is the data in the X-ray range which sets constraints on the baryonic loading.

While our results apply to PKS B1424-418 specifically, we emphasize the importance of a self-consistent description of the SED as opposed to to generic approaches relating the gamma-ray and neutrino luminosities. The studied blazar serves as a counter-example for the direct correlation of these, which means that this assumption does not hold in general and has to be used with care. We have also presented an example in a quantitative way on how the neutrino observation, even with one candidate event, is able to break the degeneracies of blazar models.

\section{References}

Gao, S., Pohl, M., \& Winter, W. 2016, ApJ, in press, arXiv:1610.05306

Ghisellini, G., Righi, C., Costamante, L., \& Tavecchio, F. 2017, M.N.R.A.S, 469, 255

Hümmer, S., Rüger, M., Spanier, F., \& Winter, W. 2010, Astrophys.J., 721, 630

IceCube Collaboration. 2013, Science, 342, 1242856

Kadler, M., Krauß, F., Mannheim, K., et al. 2016, Nature Physics, 12, 807

Vurm, I., \& Poutanen, J. 2009, Astrophys.J., 698, 293 